\title{
BOOK METADATA FOR AN OPEN ACCESS WORLD

\section{ABSTRACT}

Most book and monograph publications still lack visibility within an electronic environment due to missing digitalized and persistent bibliographic data and/or fragmentary portability or interoperability based on the variety of formats, resulting in a more error-prone, non-standardized communication between various indexing systems and platforms. But books that cannot be discovered will not be But OA metadata for books is essential for the read. OA metadata for books is essential for the transformation of the whole scholarly landscape, and one of its greatest advantages is full, immediate accessibility. ${ }^{1}$

The point of origin for the OA META project was the realization that available book metadata (i.e. ONIX/MARC records) was not primarily created for the usage of indexing within an electronic research environment. Consequentially, both detail and focus of elements tagged in datasets are not necessarily selected to output solidified and persistent book records. The question was how to enrich or edit available metadata so that it can unfold its greatest impact in an e-context.

\section{OBJECTIVE}

ScienceOpen is creating a metadata hub with a free interface to create, maintain or (formatspecifically) enrich, and export available OA book metadata that allows publishers to create comprehensive, interoperable metadata readily integrable with persistent IDs and transferable between systems to connect to the infrastructure of the scholarly publishing landscape more easily to increase visibility, discoverability, and distribution of OA metadata for books and monographs-all openly licensed and easily accessible, in compliance with common standards and best practices.

\section{METHOD}

Current common tagging practices of use cases from a variety of format samples were analyzed to identify problematic data fields not satisfactorily covered in a specific format and areas where tagging conventions are not yet fully established and thus lead to various possible tagging practices.
For immediate feedback on current metadata standards in use we organized a workshop with the divers stakeholders from the German publishing industry with special focus on said problematic data fields and missing elements, primary data objectives, and required operability between systems. Surveys were used to gather input on data and usage standards throughout the publishing landscape.

All results were gathered in an element mapping sheet building upon the crosswalk table from ONIX 3.0 to MARC 21 by Godby ${ }^{2}$ with revisions based on sample files by a range of (OA and non-OA) publishers and the direct input from the Stakeholder workshop.

\section{FINDINGS}

Overall notable was the predominance of MARC 21 and ONIX records as neither is necessarily used to tag relevant elements in a persistent format that readily allows for an aggregation of different book format records or to attribute the required detail for rich indexing within an e-environment (esp. persistent IDs such as DOIs and ORCIDs, or self-uris and access permissions for e-versions). The most surprising finding from the surveys was, however, that BITS XML was hardly recognized as an appropriate standard in comparison to the wellestablished and much more prevalent JATS format equivalent from article publishing:

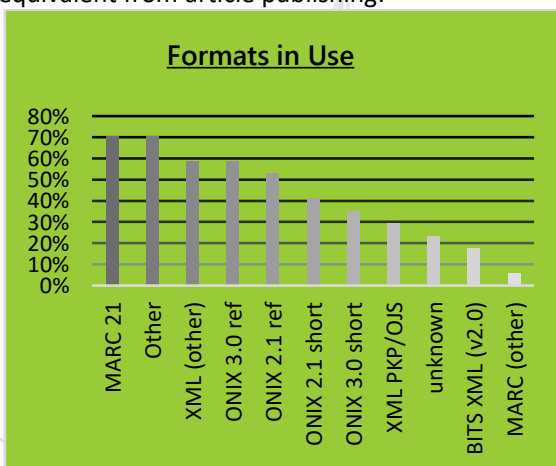

As the greatest drawback was perceived that data conversion from ONIX is missing a link to easily bring records into a Crossref-compatible form due to the discrepancy of a primarily paper-based publication workflow in contrast to electronicbased records.

The overall assessment revealed a gap in terms of data format/management when it comes to indexing content with the aim to create a unified record presence within databases, repositories, or research search engines.

\section{RESULTS}

The findings and feedback were collected and used to define a core set of metadata that will be stored within the database, and which can be enriched and outputted according to format-specific requirements via a user interface. The results were combined in a mapping table of ONIX/MARC/BITS elements marking those that need to be stored in the database to keep the record viable according to form-specific requirements, and those that require and allow for a direct translation. Requirements for outputs in MARCXML were noted but based on input from the stakeholder workshop there was no immediate interest to input MARC records. The vast majority of stakeholders were looking for an input of ONIX 3.0 records with an output of Crossref compatible XML. The project work to-date has therefore focused on ONIX XML metadata and following input/output translations:

Required Input Formats for OA META Beta Platform

- ONIX 2.1+3.0 (short + reference)

- BITS XML

Crossref record

No MARC input
$\quad$ result Stakeholder Workshop

Required Output Formats

- $\quad$ BITS (= NLM/JATS-compatible standard)

- ONIX 3.0 ref

MARCXML

To illustrate the initial mapping stages, the nex figure is showing common ONIX data variants for $O A$ license information and its equivalent in BITS $X M L$ presenting one of the potentially problematic areas in terms of data matching:

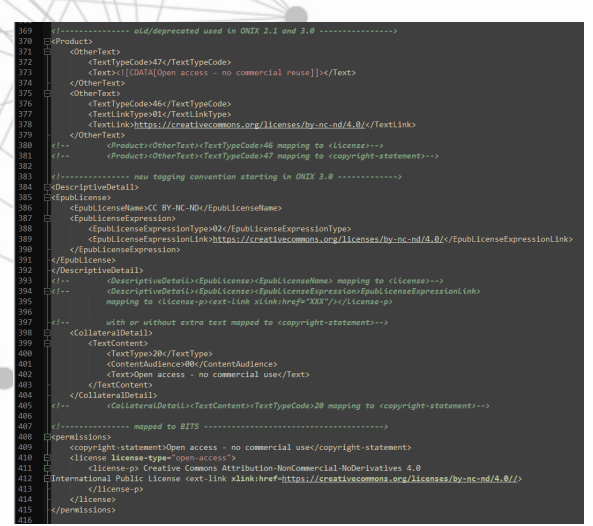

The ScienceOpen interface shall thus be used to help solidify and standardize relevant metadata elements for usage in an e-environment.

\section{CONCLUSION}

With this project we hope to support particularly smaller and mid-sized publishers that might have more restricted capacities with regard to technical infrastructure, know-how, or workforce to enable them to create rich, interoperable metadata in compliance with common standards and best practices, by providing them with the means to freely and easily update records with persistent IDs and to enhance them via a user interface so they become transferable between systems to connect to the infrastructure of the scholaly publishing landscape. With this increase in visibility, discoverability, and distribution of OA metadata for books and monographs we hope to contribute in the development towards a more open and inclusive scientific landscape.

\section{REFERENCES}

Antonia Schrader, David Böhm, Alexander Grossmann Michael Reiche "Einleitung" OpenAccess-Publikationsworkflow für a kademische Bücher: Ein Handbuch für Hochschulen und Universitäten (2020): 19-31 doi: $10.33968 /$ 9783966270175-00.

2. Carol Jean Godby. 2012. A Crosswalk from ONIX Version 3. Ofor Books to MARC 21. Dublin, Ohio: OCLC Research. Report and crosswalk. 